\section{A confirmation of chronic graft-versus-host disease prediction using allogeneic HY antibodies following sex-mismatched hematopoietic cell transplantation}

Chronic graft-versus-host disease (cGvHD) limits the effectiveness of hematopoietic cell transplantation (HCT) and often leads to significant morbidity and non-relapse mortality. ${ }^{1}$ We have previously shown that antibodies targeting Y-chromosome encoded proteins (HY antibodies) detected at 3-months post transplant in male HCT patients who receive grafts from a female donor $(F \rightarrow M)$ are associated with $\mathrm{cGvHD}$ development, non-relapse mortality (NRM), and decreased overall survival (OS)., However, it is not known if this marker can be applied to the general population. In this multi-center, retrospective cohort study, we measured HY antibody levels in 234 $\mathrm{F} \rightarrow \mathrm{M} \mathrm{HCT}$ patients with either plasma or serum samples collected at both the 3-month and 1-year post-transplant time points and determined the multivariable-adjusted relationship between the presence of multiple HY antibodies (represented as HY score) and cGvHD development.

We quantified antibodies against the five most inform-

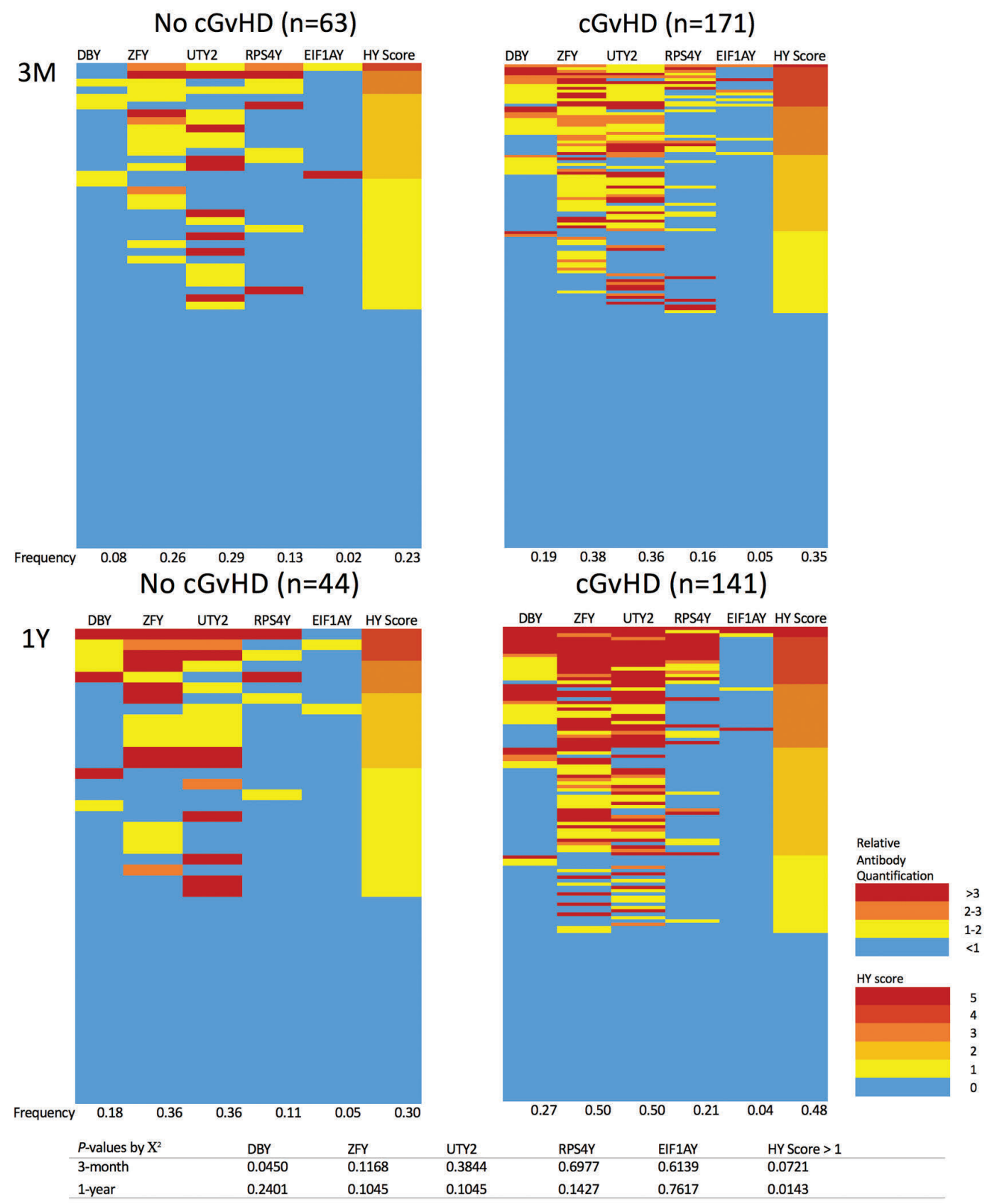

Figure 1. Anti-HY antibody responses in female donor to male recipient $(\mathrm{F} \rightarrow \mathrm{M})$ hematopoietic cell transplantation (HCT) recipients with and without chronic graft-versus-host disease (cGvHD). Comparison included $234 \mathrm{~F} \rightarrow \mathrm{M}$ samples taken three months post transplant (3M) and 185 samples taken one year post transplant (1Y). Intensity of the antibody response is color-coded as a multiple of each HY-seropositivity threshold. HY score represents the cumulative number of seropositive HY antigens. Seropositivity frequencies for each antibody are listed along the bottom border of each heat map. HY score frequency refers to the frequency of an HY score $>1$. 
ative HY antigens (DBY, UTY2, ZFY, RPS4Y, and EIF1AY) in sera samples from 79 Blood and Marrow Transplant Clinical Trials Network (BMT CTN) subjects and plasma samples from 155 Dana Farber Cancer Institute (DFCI) patients who underwent $\mathrm{F} \rightarrow \mathrm{M}$ transplantation between 2004 and 2014 (Table 1). ${ }^{2,4}$ The trials from which these data were taken used cGvHD development as their clinical end points. To set HY antibody seropositivity thresholds, a control group of 60 paired sera and EDTA plasma samples collected from healthy male blood donors was obtained from the National Heart, Lung and Blood Institute (NHLBI) BioLINCC repository (REDS II-LAPS study). Antibody detection and threshold calculation was performed according to our previously published protein microarray platform. ${ }^{5}$ Full details of methods and statistical analyses can be found in the Online Supplementary
Appendix. There was no appreciable difference in seropositivity cutoffs and antibody quantifications between plasma and sera samples (Online Supplementary Figure S1).

Our 3-month univariate analysis of $234 \mathrm{~F} \rightarrow \mathrm{M}$ patients showed antibodies against DBY had a 2.3-fold higher relative frequency in the $171 \mathrm{cGvHD}$ patients than in the 63 non-cGvHD patients $(P=0.045)$. The frequency of $\mathrm{HY}$ seropositivity was higher in future cGvHD patients across all five antigens. As nobody has cGvHD at 3months post transplant, this measurement is predictive of cGvHD development 6-12 months later. National Institutes of Health (NIH) cGvHD severity (mild/moderate/severe) was available for the 79 BMT-CTN clinical trial patients. Again, DBY seropositivity at three months was most predictive of severe cGvHD $(P=0.021)$. The

Table 1. Patients' characteristics

\begin{tabular}{|c|c|c|c|}
\hline & $\begin{array}{c}\text { Any HY seropositivity 3-months } \\
\text { post HCT (\%) }\end{array}$ & $\begin{array}{l}\text { Seronegativity 3-months } \\
\text { HY post HCT (\%) }\end{array}$ & $P$ \\
\hline Total cohort & $118(50.5 \%)$ & $116(49.5 \%)$ & \\
\hline Median patient age, y (range) & $48(11-69)$ & $47(5-73)$ & 0.577 \\
\hline Median donor age, y (range) & $41(15-65)$ & $40(15-70)$ & 0.323 \\
\hline Sample type & & & 0.431 \\
\hline Plasma + EDTA & $103(87.3 \%)$ & $96(82.8 \%)$ & \\
\hline Serum & $15(12.7 \%)$ & $20(17.2 \%)$ & \\
\hline Primary disease & & & 0.089 \\
\hline AML & $44(37.3 \%)$ & $41(35.3 \%)$ & \\
\hline ALL & $22(18.6 \%)$ & $23(19.8 \%)$ & \\
\hline MDS & $9(7.6 \%)$ & $19(16.4 \%)$ & \\
\hline CML & $6(5.1 \%)$ & $10(8.6 \%)$ & \\
\hline Lymphoma & $24(20.3 \%)$ & $9(7.8 \%)$ & \\
\hline CLL, SLL, PLL & $9(7.6 \%)$ & $10(8.6 \%)$ & \\
\hline Others & $4(3.4 \%)$ & $4(3.5 \%)$ & \\
\hline CMV seropositivity & & & 0.250 \\
\hline $\mathrm{D}+\mathrm{R}+$ & $31(26.3 \%)$ & $31(26.7 \%)$ & \\
\hline $\mathrm{D}+\mathrm{R}-$ & $25(21.2 \%)$ & $15(12.9 \%)$ & \\
\hline $\mathrm{D}-\mathrm{R}+$ & $27(22.9 \%)$ & $37(31.9 \%)$ & \\
\hline D- R- & $35(29.7 \%)$ & $33(28.4 \%)$ & \\
\hline Donor type & & & 0.146 \\
\hline MRD & $64(54.2 \%)$ & $51(44.0 \%)$ & \\
\hline MUD & $41(34.7 \%)$ & $57(49.1 \%)$ & \\
\hline MMRD & $1(0.8 \%)$ & $1(0.9 \%)$ & \\
\hline MMUD & $12(10.2 \%)$ & $7(6.0 \%)$ & \\
\hline Cell source & & & 0.111 \\
\hline Bone marrow & $11(9.3 \%)$ & $20(17.2 \%)$ & \\
\hline Peripheral blood & $107(90.7 \%)$ & $96(82.8 \%)$ & \\
\hline Conditioning & & & 0.241 \\
\hline With ATG & $4(3.4 \%)$ & $9(7.8 \%)$ & \\
\hline Without ATG & $114(96.6 \%)$ & 107 (92.2\%) & \\
\hline aGvHD & & & 0.086 \\
\hline Yes & $57(48.3 \%)$ & $70(60.3 \%)$ & \\
\hline No & $61(51.7 \%)$ & $46(39.7 \%)$ & \\
\hline
\end{tabular}

HCT: hematopoietic cell transplantation; AML: acute myeloid leukemia; ALL: acute lymphoblastic leukemia; MDS: myelodysplastic syndrome; CML: chronic myelogenous leukemia; CLL: chronic lymphocytic leukemia; SLL: small lymphocytic leukemia; PLL: prolymphocytic leukemia; CMV: cytomegalovirus; MRD: matched related donor; MUD: matched unrelated donor; MMRD: mismatched related donor; MMUD: mismatched unrelated donor; ATG: anti-thymocyte globulin; aGvHD: acute graft-versus-host disease. 
cumulative number of HY antibody seropositivities for a given sample is reported as the HY score, which we showed in previous papers to be the most robust predictor of $\mathrm{cGvHD}$ development. ${ }^{2}$ An HY score $>1$ at three months predicted for cGvHD severity $(P=0.039)$ (Online Supplementary Figure S2).

Multivariable analysis of 3-month measurements, adjusted for patient age, donor age, disease, donor relation, cell source, data source, and acute GvHD (aGvHD) grade, shows increased HY score associated with increased cGvHD risk (Table 2). An HY score $>2$ proved to be the best predictor of cGvHD development (OR 3.63, $P=0.027)$. The presence of anti-DBY antibodies at three months also trended towards $\mathrm{cGvHD}$ development (OR 2.78, $P=0.053$ ), confirming that HY score is a powerful marker for predicting future cGvHD development in $\mathrm{F} \rightarrow \mathrm{M}$ HCT patients.

Of the 234 patients, there were 185 patients who survived one year. Forty-four never developed cGvHD, while 141 developed cGvHD at any time. A 1-year HY score $>1$ associated with a 1.6-fold increase in cGvHD development by univariate analysis $(P=0.029)$. One-year HY score also tended to associate with moderate or severe cGvHD $(P=0.056)$ (Online Supplementary Figure S2). One-year HY score in a multivariable analysis did not show any association with cGvHD (Table 2). We believe that the 1-year HY association with $\mathrm{cGvHD}$ is diminished from the 3-month post-HCT measurement due either to earlier death or to corticosteroid treatment of cGvHD before the 1-year measurement. In support of this theory, we found that prior aGvHD development with corticosteroid treatment was an effect modifier of the association between HY antibodies at one year and cGvHD development (Online Supplementary Table S1). Furthermore, other cGvHD therapies such as antithymocyte globulin (ATG) and rituximab have been shown to reduce antibody levels. ${ }^{6}$ Considering cGvHD treatment confounds the detection of HY antibodies at 1-year, we believe 3-month HY antibodies are both predictive and more sensitive markers for $\mathrm{cGvHD}$ risk than levels at one year.

In this study, 3-month HY score did not predict for other clinical outcomes, including NRM, OS, and relapse (Online Supplementary Table S3). However, 1-year HY score associated with increased OS, decreased NRM, and decreased relapse, even after multivariable adjustment (Online Supplementary Table S3). Moreover, cGvHD development was similarly associated with increased OS, decreased NRM, and decreased relapse (Online Supplementary Figure S3). While decreased relapse is expected with alloantibody development due to a beneficial graft-versus-leukemia effect in addition to the graft-versus-host response, both our previous studies and the established literature have shown that cGvHD development is associated with increased NRM and decreased OS. ${ }^{2,7,8}$ The primary end point of the contributing BMTCTN studies was cGvHD development, providing high diagnostic confidence. It is not clear why NRM did not associate with cGvHD development in this patient cohort. However, 3-month HY score strongly predicts cGvHD development, the primary trial end point.

There are several other limitations to this study. First, due to lack of data, our regression model did not include prior B-cell depleting therapy, nor could it distinguish between patients with B- versus $\mathrm{T}$-cell malignancies. Second, the HY score is a simple summation of seropositivity, when in reality DBY appears to be the largest driver of the HY score-cGvHD relationship. Future models could weigh individual HY antigens differently in the HY score calculation, but the purpose of this study was to
Table 2. Multivariable analyses for the impact of HY antibody seropositivity on CGvHD development.

CGVHD
$0 R^{*}(95 \%$ CI)

\section{Months}

HY Score 3M

As a group variable
$0-1$

2

3-5

DBY alone

UTY2 alone

ZFY alone

RPS4Y alone

EIF1AY alone

1

$1.20(0.54-2.70)$

3.63 (1.16-11.4)

0.66

$2.78(0.99-7.80)$

0.027

$1.37(0.72-2.64)$

0.053

0.34

0.09

$1.82(0.91-3.65)$

0.37

1 Year

HY Score $1 Y$

As a group variable

0-1

2

$3-5$

$1.49(0.63-3.55)$

0.61 ref

37

$\begin{array}{lll}\text { DBY alone } & 1.77(0.71-4.39) & 0.22 \\ \text { UTY2 alone } & 1.52(0.72-3.20) & 0.27 \\ \text { ZFY alone } & 1.62(0.76-3.44) & 0.21 \\ \text { RPS4Y alone } & 2.19(0.75-6.42) & 0.15 \\ \text { EIF1AY alone } & 057(0.098-3.29) & 0.53\end{array}$

*Adjusted for patient age, donor age, disease, donor relation, cell source, data source, and acute graft-versus-host disease (aGvHD) grade. cGvHD: chronic graftversus-host disease; NRM, non-relapse mortality; OS: overall survival; OR: odds ratio; CI: confidence interval.

confirm that the aggregate sum of HY seropositivities was a reliable predictor of cGvHD risk. Lastly, this study did not have sufficient data on NIH severity or cGvHD organ scores to adequately power an in-depth analysis of the association of the HY score with cGvHD severity or organ involvement. These are all areas we hope to address in future studies.

A common critique of the HY model is that the clinical utility of measuring $\mathrm{HY}$ antibodies is limited to a subset of HCT patients. While $\mathrm{F} \rightarrow \mathrm{M}$ transplant recipients only constitute $25 \%$ of all HCT patients, in addition to prognostic value for these patients, the HY system also provides a research model for determining the role of $\mathrm{B}$ cells in the cGvHD disease process and studying future cGvHD therapies. Studies that show allogeneic antibody deposition in cGvHD tissues imply a pathological role of alloantibodies in cGvHD. ${ }^{9}$ We believe HY antibodies may reflect overall alloantibody development. The predictive value of 3-month HY antibody levels has identified a Bcell role in the pathogenesis of cGvHD, B-cell depleting agents such as rituximab and ibrutinib are effective treatments of cGvHD. ${ }^{10,11}$

In summary, this multi-center study of HY antibodies measured in a blinded fashion confirms that $\mathrm{HY}$ antibodies detected at the 3-month post-transplant time point in $\mathrm{F} \rightarrow \mathrm{M} \mathrm{HCT}$ patients predict $\mathrm{CGvHD}$ development. The 3month HY score has its greatest utility in predicting which patients are more likely to develop severe cGvHD. As such, HY scoring at three months could identify patients at high risk for cGvHD prevention trials. 
Jed Paul, ${ }^{1}$ Hideki Nakasone, ${ }^{2}$ Bita Sahaf, Fang Wu, ${ }^{1}$ Kathy Wang, ${ }^{3}$ Vincent Ho, ${ }^{3}$ Juan Wu ${ }^{4}{ }^{4}$ Haesook Kim, ${ }^{3}$ Bruce Blazar, Jerome Ritz, ${ }^{3}$ Alan Howard, ${ }^{6}$ Corey Cutler, ${ }^{3}$ and David Miklos

'Division of Blood and Marrow Transplantation, Stanford University School of Medicine, Stanford, CA, USA; ${ }^{2}$ Division of Hematology, Saitama Medical Center, Jichi Medicial University, Saitama, Japan; ${ }^{3}$ Division of Hematological Malignancies, DanaFarber Cancer Institute, Boston, MA, USA; ${ }^{4}$ The Emmes Corporation, Rockville, MD, USA; ${ }^{5}$ Division of Pediatrics BMT, Minneapolis, MN, USA and ${ }^{6}$ Center for International Blood and Marrow Transplant Research, Minneapolis Campus, Minneapolis, MN, USA

Funding: this work was supported by National Institutes of Health, National Cancer Institute grants P01CA142106, CA183559, and CA183560, as well as by an ancillary laboratory study award provided by the Blood and Marrow Transplant Clinical Trials Network and the National Heart, Lung and Blood Institute and the National Cancer Institute (\#U10HLO69294).

Acknowledgments: we also acknowledge the BMT CTN 0201 and 0402 study investigators and participating centers.

Support for these studies was provided to the Blood and Marrow Transplant Clinical Trials Network by grant \#U10HL069294 from the National Heart, Lung and Blood Institute and the National Cancer Institute. The Department of the Navy, Office of Navy Research and the National Marrow Donor Program also provided support for the BMT CTN 0201 study. BMT CTN 0402 biospecimens were obtained from the NHLBI Biologic Specimen and Data Repository Information Coordinating Center (BioLINCC). Enrollment support was provided by DKMS Germany. Any views, opinions, findings, conclusions or recommendations expressed in this material are those of the author(s) and do not reflect the views or the official policy or position of the above-mentioned parties.

This manuscript was prepared using REDS II-LAPS Research Materials obtained from the NHLBI Biologic Specimen and Data Repository Information Coordinating Center and does not necessarily reflect the opinions or views of the REDS II-LAPS or the NHLBI. $H N$ was a recipient of a grant from Takeda Science Foundation.
Correspondence: DAVID B. MIKLOS.

dmiklos@stanford.edu

doi:10.3324/haematol.2018.199646

Information on authorship, contributions, and financial \& other disclosures was provided by the authors and is available with the online version of this article at www. haematologica.org.

\section{References}

1. Lennard AL, Jackson GH. Stem cell transplantation. West J Med. 2001;175(1):42-46.

2. Nakasone H, Tian L, Sahaf B, et al. Allogeneic HY antibodies detected 3 months after female-to-male HCT predict chronic GVHD and nonrelapse mortality in humans. Blood. 2015;125(20):3193-3201.

3. Miklos DB, Kim HT, Zorn E, et al. Antibody response to DBY minor histocompatibility antigen is induced after allogeneic stem cell transplantation and in healthy female donors. Blood. 2004;103(1):353-359.

4. Miklos DB, Kim HT, Miller KH, et al. Antibody responses to H-Y minor histocompatibility antigens correlate with chronic graft-versus-host disease and disease remission. Blood. 2005;105(7):29732978

5. Paul J, Sahaf B, Perloff S, et al. High-throughput allogeneic antibody detection using protein microarrays. J Immunol Methods. 2016; 432:57-64.

6. Clatworthy MR. Targeting B Cells and Antibody in Transplantation. Am J Transplant. 2011;11(7):1359-1367.

7. Bhatia S, Francisco L, Carter A, et al. Late mortality after allogeneic hematopoietic cell transplantation and functional status of long-term survivors: report from the Bone Marrow Transplant Survivor Study. Blood. 2007;110(10):3784-3792

8. Lee SJ, Klein JP, Barrett AJ, et al. Severity of chronic graft-versus-host disease: association with treatment-related mortality and relapse. Blood. 2002;100(2):406-414.

9. Jin $\mathrm{H}, \mathrm{Ni} \mathrm{X}$, Deng R, et al. Antibodies from donor B cells perpetuate cutaneous chronic graft-versus-host disease in mice. Blood. 2016; 127(18):2249-2260

10. Arai S, Sahaf B, Narasimhan B, et al. Prophylactic rituximab after allogeneic transplantation decreases B-cell alloimmunity with low chronic GVHD incidence. Blood. 2012;119(25):6145-6154.

11. Miklos D, Cutler CS, Arora M, et al. Ibrutinib for chronic graft-versus-host disease after failure of prior therapy. Blood. 2017; 130(21):2243-2250 